Full length article

\title{
Treatment with galectin- 1 eye drops regulates mast cell degranulation and attenuates the severity of conjunctivitis
}

\author{
Claudia Mello-Bosnic ${ }^{\mathrm{a}}$, Alexandre Dantas Gimenes ${ }^{\mathrm{b}}$, Sonia Maria Oliani ${ }^{\mathrm{a}}$, Cristiane Damas Gil ${ }^{\mathrm{a}, \mathrm{b}, *}$ \\ a UNESP - Universidade Estadual Paulista, Programa de Pós-Graduação em Biociências, Instituto de Biociências, Letras e Ciências Exatas, São José do Rio Preto, SP, Brazil \\ ${ }^{\mathrm{b}}$ UNIFESP - Universidade Federal de São Paulo, Departamento de Morfologia e Genética, São Paulo, SP, Brazil
}

\section{A R T I C L E I N F O}

\section{Keywords:}

Compound 48/80

Eosinophil

Eye

Mitogen-activated protein kinases

Inflammation

\begin{abstract}
A B S T R A C T
Galectin-1 (Gal-1) is a $\beta$-galactoside-binding protein with diverse biological activities in the pathogenesis of inflammation, however the mechanisms by which Gal-1 modulates cellular responses in allergic inflammatory processes have not been fully determined. In this study, we evaluated the therapeutic potential of Gal-1 eye drops in an experimental model of conjunctivitis. Wistar rats received a topical application of compound (C)48/ $80(100 \mathrm{mg} / \mathrm{ml})$ into right eyes and a drop of vehicle into the contralateral eye. Another group of rats received Gal-1 ( 0.3 or $3 \mu \mathrm{g}$ /eye) or sodium cromoglycate (SCG; $40 \mathrm{mg} / \mathrm{ml}$ ) in both eyes and, after $15 \mathrm{~min}$, right eye was challenged with $\mathrm{C} 48 / 80$. Conjunctivitis-induced by $\mathrm{C} 48 / 80$ was characterized by severe eyelid oedema and tearing, but clinical signs were ameliorated by eye drop doses of both Gal-1 (0.3/3 $\mu \mathrm{g})$ and SCG. As expected, an increased proportion of degranulated mast cells $(62 \%, P<0.01)$ and lower histamine levels were observed after $6 \mathrm{~h}$ of C48/80 challenge, compared to control (32\%). This effect was abrogated by Gal-1 and SCG, which reduced mast cell degranulation (31-36\%), eosinophil migration and eosinophil peroxidase levels in the eyes. Gal-1 $(3 \mu \mathrm{g})$ and SCG treatments also decreased IL-4 levels, as well as activation of mitogen activated protein kinases compared to untreated C48/80 eyes. Our findings suggest that Gal-1 eye drops represent a new therapeutic strategy for ocular allergic inflammation.
\end{abstract}

\section{Introduction}

Pharmacological treatment of ocular inflammatory processes, especially allergic conjunctivitis (AC), includes antihistamines, mast cell membrane stabilizers, non-steroidal anti-inflammatory drugs and corticosteroids. The latter is used in the more severe AC and has higher risk of adverse effects, such as increased intraocular pressure and cataracts (Leonardi et al., 2006). Therefore, the investigation of new pharmacological agents that are more effective in controlling the inflammatory response with fewer adverse effects, is fundamental. We highlight galectin-1 (Gal-1), a $14.5 \mathrm{kDa} \beta$-galactoside-binding protein that regulates the inflammatory process, as a potential anti-inflammatory agent (Norling et al., 2009).

Gal-1 belongs to a family of proteins, initially referred to as S-type lectins, that share a highly-conserved carbohydrate-recognition domain (CRD) that is responsible for their high affinity for $\beta$-galactosides in glycoconjugates (Leffler et al., 2002). In this regard, Gal-1 recognizes multiple galactose- $\beta 1-4-\mathrm{N}$-acetyl-glucosamine (N-acetyl-lactosamine [LacNAc]) units present on the branches of $\mathrm{N}$ - or O-linked glycans on diverse cell surface receptors, such as CD45, CD43, CD69, and vascular endothelial growth factor receptor 2 (VEGFR2) (Kanda et al., 2015; Sundblad et al., 2017). By interacting with a variety of glycosylated receptors, this lectin translates glycan-containing information into regulatory programs that control immune cell homeostasis (Sundblad et al., 2017). Additionally, Gal-1 expression has been detected in several cells related to the inflammatory response, especially neutrophils, mast cells, macrophages, lymphocytes and endothelial cells, suggesting an important role in the generation and maintenance of immunological tolerance (Blaser et al., 1998; Gil et al., 2006a, 2006b; La et al., 2003; Rabinovich et al., 1998; Zuñiga et al., 2001).

The anti-inflammatory role of Gal-1 has been demonstrated in in vitro and in vivo experimental models after exogenous administration of recombinant Gal-1. In vitro, incubation with Gal-1 inhibited the migration of human neutrophils and lymphocytes through endothelial cells after inflammatory stimulation with interleukin (IL)-8 or tumour necrosis factor- $\alpha$ (TNF- $\alpha$ ), respectively (La et al., 2003; Norling et al., 2008). In vivo, pharmacological treatment with Ga-1 regulates the inflammatory response by inhibiting leukocyte transmigration and release of proinflammatory cytokines such as TNF- $\alpha$, interferon- $\gamma$ (IFN- $\gamma$ ), IL-1ß, IL-2, IL-6 and IL-12 ( Gil et al., 2006a, 2011; La et al., 2003;

\footnotetext{
* Correspondence to: Departamento de Morfologia e Genética, UNIFESP - Universidade Federal de São Paulo, Rua Botucatu 740, Ed. Lemos Torres - $3^{\circ}$ andar, 04023-900 São Paulo, São Paulo, Brazil.

E-mail address: cristiane.gil@unifesp.br (C.D. Gil).
} 
Rabinovich et al., 1999; Santucci et al., 2003; Toscano et al., 2006; Zanon et al., 2015).

Previously, we demonstrated the inhibitory effect of Gal-1 in an experimental model of ovalbumin (OVA)-induced AC in mice, on the production of Th2 cytokines (IL-4, IL-13) and eotaxin by lymph nodes. This was associated with decreased clinical disease signs and production of anti-OVA IgE (Mello et al., 2015). However, the mechanisms by which Gal-1 modulates cellular responses in allergic inflammatory processes have not been fully determined, particularly in ocular models.

Compound $48 / 80(\mathrm{C} 48 / 80)$ is a polymer produced by the condensation of N-methyl-p-methoxyphenethylamine with formaldehyde and acts directly on G-proteins to produce mast cell degranulation, one of the major effector cells that contributes for the development of the acute manifestations of allergy (Elieh Ali Komi et al., 2018; Papathanassiou et al., 2011; Tiligada et al., 2000; Udell and Abelson, 1981). Topical application of C48/80 is able to produce the signs and symptoms of ocular allergy (itching, injection, chemosis, and mucous discharge) and represents a useful tool to evaluate the effects of therapeutic agents in the ocular allergy (Udell and Abelson, 1981).

In view of these considerations and the need for safer therapies for the treatment of conjunctivitis and other allergic inflammatory processes (Ackerman et al., 2016; Leonardi et al., 2006), we evaluate the effect of treatment with Gal-1 eye drops in an experimental model of C48/80-induced conjunctivitis in rats.

\section{Materials and methods}

\subsection{Animals}

Wistar rats, weighing 200-250 g, were housed in a $12 \mathrm{~h}$ light-dark cycle and were allowed food and water ad libitum. All experimental procedures were submitted to and approved by the Ethics Committee in Animal Experimentation of the São Paulo State University (CEUA 092/ 2014).

\subsection{Compound 48/80-induced conjunctivitis and treatment protocols}

The application of the mast cell secretagogue compound 48/80 $(\mathrm{C} 48 / 80)$ to rat, mouse and rabbit eyes has been reported to induce histologic and clinical changes resembling those seen in patients with AC, suggesting that a model of ocular anaphylaxis could be clinically relevant (Bucolo et al., 1993; Bundoc and Keane-Myers, 2007; Ko et al., 2000; Papathanassiou et al., 2011; Tiligada et al., 2000). For our study, Wistar rats were distributed into groups of 7-10 animals per treatment, as indicated in Table 1. Experimental conjunctivitis was induced in group I by a topical application of a single $20 \mu \mathrm{l}$ drop of C48/80 (Sigma Chemical Company, St Louis, MO, USA) at $100 \mathrm{mg} / \mathrm{ml}$ (Papathanassiou et al., 2011; Tiligada et al., 2000), diluted in PBS, onto the conjunctival sac. As a control, the contralateral eye (left) received only PBS. Groups II, III and IV were administered with a $20 \mu \mathrm{l}$ topical drop of $0.3,3 \mu \mathrm{g}$ / eye of Gal-1 or $40 \mathrm{mg} / \mathrm{ml}$ of mast cell stabilizer sodium cromoglycate (SCG), respectively, and after $10 \mathrm{~min}, \mathrm{C} 48 / 80$ (experimental groups) or PBS (control groups). Doses of C48/80, Gal-1 and SCG were scaled up

Table 1

Experimental conjunctivitis induced in rats and respective treatments.

\begin{tabular}{lll}
\hline Groups & Right eye (Experimental) & Left eye (Control) \\
\hline I & C48/80 $(100 \mathrm{mg} / \mathrm{ml})$ & PBS \\
II & Gal-1 $(0.3 \mu \mathrm{g} / \mathrm{eye})+\mathrm{C} 48 / 80(100 \mathrm{mg} / \mathrm{ml})$ & Gal-1 $(0.3 \mu \mathrm{g} / \mathrm{eye})+$ PBS \\
III & Gal-1 $(3 \mu \mathrm{g} / \mathrm{eye})+\mathrm{C} 48 / 80(100 \mathrm{mg} / \mathrm{ml})$ & Gal- $1(3 \mu \mathrm{e} / \mathrm{eye})+\mathrm{PBS}$ \\
IV & SCG $(40 \mathrm{mg} / \mathrm{ml})+\mathrm{C} 48 / 80(100 \mathrm{mg} / \mathrm{ml})$ & SCG $(40 \mathrm{mg} / \mathrm{ml})+$ PBS
\end{tabular}

A single $20 \mu$ drop of each agent was instilled into the lower conjunctival fornix of one eye, at $10 \mathrm{~min}$ intervals. The contralateral eye was used as the respective control ( $\mathrm{n}=4-7)$. C48/80, compound 48/80; Gal-1, galectin-1; SCG, sodium cromoglycate; PBS, phosphate-buffered saline. from previous studies (Gil et al., 2006a; Mello et al., 2015; Papathanassiou et al., 2011). All drug instillations on rat conjunctival sacs were performed in animals anesthetised with ketamine and xylazine.

After 20 min of C48/80 challenge, rats were clinically examined to verify the occurrence and severity of conjunctivitis. Four clinical signs were observed: chemosis, conjunctival hyperaemia, lid oedema and tearing. Scoring similar to that described by Magone et al. (1998) was performed and each parameter was graded on a scale ranging from 0 to $3+(0=$ absence; $1=$ mild; $2=$ moderate and $3=$ severe symptoms $)$. Thus, each animal received a total clinical score ranging from 0 to $12+$, and the data were expressed as the mean \pm standard error of the mean (S.E.M.) for each group. Six $\mathrm{h}$ after the last C48/80 challenge, animals were euthanized, and the eyes and eyelids were collected.

\subsection{Histamine and Th1/Th2 cytokine levels}

Histamine and cytokine levels were determined in eyes from rats subjected to different experimental conditions. Eye samples were sonicated in a $50 \mathrm{mM}$ Tris- $\mathrm{HCl}, 150 \mathrm{mM} \mathrm{NaCl}$, and $1 \%$ Triton-X, pH 7.4 buffer containing complete protease inhibitor cocktail and PhosSTOP tablets (Roche Applied Science, Mannheim, Germany). Subsequently, samples were centrifuged at $10,000 \times g$ for $20 \mathrm{~min}$ at $4^{\circ} \mathrm{C}$ to obtain organ supernatants.

Histamine concentrations (nM) were measured using a commercially available immunoassay kit (MyBioSource, San Diego, CA) and the levels were estimated according to the manufacturer's instructions. All estimations were performed in duplicate and the data expressed as the mean \pm S.E.M.

IL-4, IL-6, IL-10 and IFN- $\gamma$ levels were tested using the MILLIPLEX MAP rat cytokine panel (RECYTMAG-65K; Millipore Corporation, USA) and MAGPIX ${ }^{\circledR}$ Multiplexing Instrument (Millipore) according to the manufacturer's instructions. The concentration of analytes was determined by MAGPIX Xponent software (Millipore Corporation, Billerica, MA, USA). All estimates were made in duplicate and the results shown as mean \pm S.E.M. of protein $(\mathrm{pg} / \mathrm{ml})$.

\subsection{Western blot analysis}

Protein levels of rat eye homogenates were determined by Bradford assay and normalized prior to boiling in Laemmli buffer (Bio-Rad Laboratories, USA). Pooled protein extracts ( $30 \mu \mathrm{g}$ per lane) of eyes ( $n=3$ animals per group) from indicated experimental conditions were loaded onto a $12 \%$ sodium dodecyl sulphate-polyacrylamide gel for electrophoresis together with appropriate molecular weight markers (Bio-Rad Life Science, USA) and transferred to ECL Hybond nitrocellulose membranes. Reversible protein staining of the membranes with $0.1 \%$ Ponceau-S in 5\% acetic acid (Santa Cruz Biotechnology) was used to verify protein transfer. Membranes were incubated for $15 \mathrm{~min}$ in 5\% BSA in Tris-buffered saline (TBS) prior to incubation with antibodies. Primary antibodies used here: mouse monoclonal anti-phosphorylated (p)-ERK 1/2 (sc-16981), anti-p-JNK (sc-6254), anti-p-p38 (sc-17852), goat polyclonal anti-eosinophil peroxidase (EPX; sc-19148) (1:200) (Santa Cruz Biotechnology) and rabbit anti-GAPDH (G9545) (1:5000) (Sigma-Aldrich), all diluted in TBS with $0.1 \%$ Tween 20. Postincubation with primary antibodies, membranes were washed for $15 \mathrm{~min}$ with TBS and subsequently incubated for $60 \mathrm{~min}$ at room temperature with the appropriate secondary antibodies. Secondary antibodies were peroxidase-conjugated rabbit anti-goat IgG, goat antirabbit (1:2000) (Thermo Fisher Scientific Inc., MI, USA) or goat antimouse (1:2000) (Millipore Corporation, CA USA). Finally, membranes were washed for $15 \mathrm{~min}$ with TBS, and immunoreactive proteins were detected (Westar Nova 2.0 chemiluminescent substrate kit; Cyanagen, Bologna, Italy) using a GeneGnome5 chemiluminescence detection system (SynGene, Cambridge, UK). Proteins were imaged and quantified using GeneTools software (SynGene) to determine the relative 
expression of indicated proteins (arbitrary units, a.u.).

\subsection{Histopathology and quantification of inflammatory cells}

Palpebral conjunctiva from rats were fixed in 4\% paraformaldehyde for $24 \mathrm{~h}$, washed in tap water, dehydrated in an increasing ethanol series, and embedded in paraffin. Sections of $4 \mu \mathrm{m}$ were obtained in a Leica RM2155 microtome (Leica Microsystems, Nussloch, Germany) and subsequently stained with haematoxylin-eosin or toluidine blue $0.5 \%$ for histopathology and inflammatory cell quantification. Leukocytes and mast cells were quantified using a $40 \times$ objective on an Axio Scope A1 Zeiss microscope (Carls Zeiss, Jena, Germany). Three semi-serial sections of conjunctivas were analysed per animal and the area was determined using AxioVision software (Carl Zeiss). Values are expressed as the mean \pm S.E.M. cells per $\mathrm{mm}^{2}$.

\subsection{Statistical analysis}

The data were analysed using one-way ANOVA followed by Bonferroni $t$-tests between corresponding time points using GraphPad Prism 4.0 software. Non-parametric analysis of the scores assigned to the conjunctival symptoms was performed using the Mann-Whitney test. In all cases, a probability value $<0.05$ was considered significant.

\section{Results}

\subsection{Topical Gal-1 eye drops regulate C48/80-induced mast cell activation in rats}

The administration of C48/80 in rat eyes induced an inflammatory response characterized by conjunctival hyperaemia and intense eyelid and conjunctiva oedema, which was abrogated by Gal-1 and SCG treatments (Fig. 1). The score confirmed the macroscopic observations, showing a significant decrease in the clinical signs in the eyes treated with Gal-1 and SCG compared to the untreated C48/80 rats (Fig. 1). No alterations were detected in the score data of the control groups.

To confirm the effect of $\mathrm{C} 48 / 80$ on mast cell activation, we performed a histological analysis of palpebral conjunctiva. Control and experimental groups presented mast cells with metachromatic cytoplasmic granules as determined by toluidine blue stain (Fig. 2A). No differences were detected in the total mast cell numbers between control and experimental groups (data not shown). However, challenge with C48/80 markedly increased the degranulated mast cell population (62\%, $P<0.01$ ) compared to the respective control group (32\%) (Fig. 2A and B). This effect was reversed by the application of Gal-1 $(0.3 \mu \mathrm{g}$ and $3 \mu \mathrm{g})$ and SCG, which resulted in a decreased mast cell degranulation $(\sim 31-36 \%)$ and an increase in intact cells (Fig. $2 \mathrm{~A}$ and B). Among the control groups I (PBS), II ( $0.3 \mu \mathrm{g} \mathrm{Gal}-1)$, III ( $3 \mu \mathrm{g} \mathrm{Gal}-1)$ and IV (SCG), there were no significant differences in the proportion of intact and degranulated cells. These findings were corroborated by the histamine levels in the eye homogenates (Fig. 2C). Administration of C48/80 (Exp. I) significantly decreased histamine levels in comparison to the control group I (Fig. 2C). In contrast, topical pre-treatment with Gal-1 and SCG reverted this effect and no differences were detected between experimental and control groups II-IV. Interestingly, eye homogenates from control groups II-IV (treated with Gal-1 or SCG only) displayed decreased levels of histamine in relation to control group I (PBS).

\subsection{Topical administration of Gal-1 reduces eosinophil influx}

The histological analysis of the eyelids of the C48/80-challenged eyes (Exp I) demonstrated an inflammatory response characterized by intense influx of eosinophils (Fig. 3A), which was abrogated by Gal-1 and SCG eye drops (Exp. II-IV). Furthermore, increased levels of eosinophil peroxidase (EPX) were detected in the eye homogenates from C48/80-challenged animals (Exp. I) compared to the others experimental groups (Fig. 3B). Quantitative analysis of eosinophils in the palpebral conjunctiva confirmed the histological and western blot findings (Fig. 3C). The C48/80-challenged group (Exp. I) produced a marked increase of cells compared to the control (group I), while the Gal-1 and SCG pharmacological treatments were effective in decreasing the influx of eosinophils.

\subsection{Effect of Gal-1 on cytokine production and Mitogen-Activated Protein kinase (MAPK) activation}

In order to verify the effect of the pharmacological treatments in the production of local cytokines we analysed eye homogenates from different experimental conditions. At the low concentration (0.3 $\mu \mathrm{g}-$ Exp.

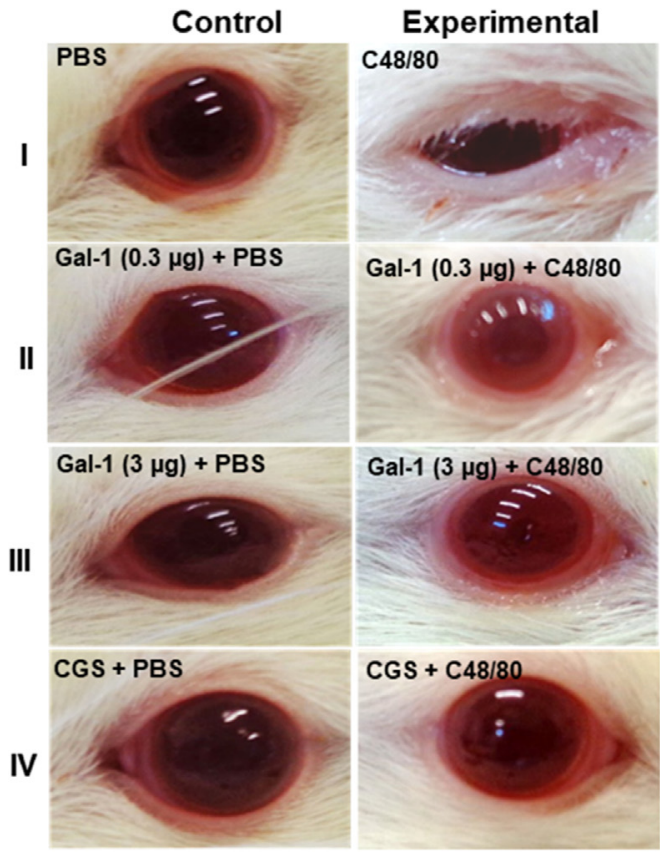

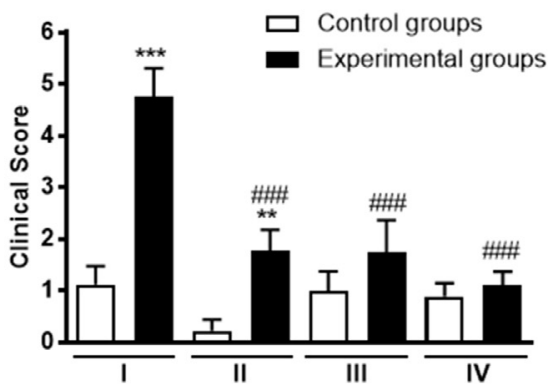

Fig. 1. Clinical score. Rat eyes observed $20 \mathrm{~min}$ after instillation of C48/80 (Experimental groups) or PBS (control groups). C48/80-challenged eyes (Experimental I) exhibited conjunctival hyperaemia and intense eyelid and conjunctiva oedema. The effect was mitigated by 0.3 and $3 \mu \mathrm{g}$ of Gal-1 (Experimental II and III) and SCG (Experimental IV) eye drops. Data represent mean \pm S.E.M. of clinical scores $(n=7-10$ animals/group). ${ }^{* * *} P<0.001$ vs. C48/80 group (Experimental I). 
A

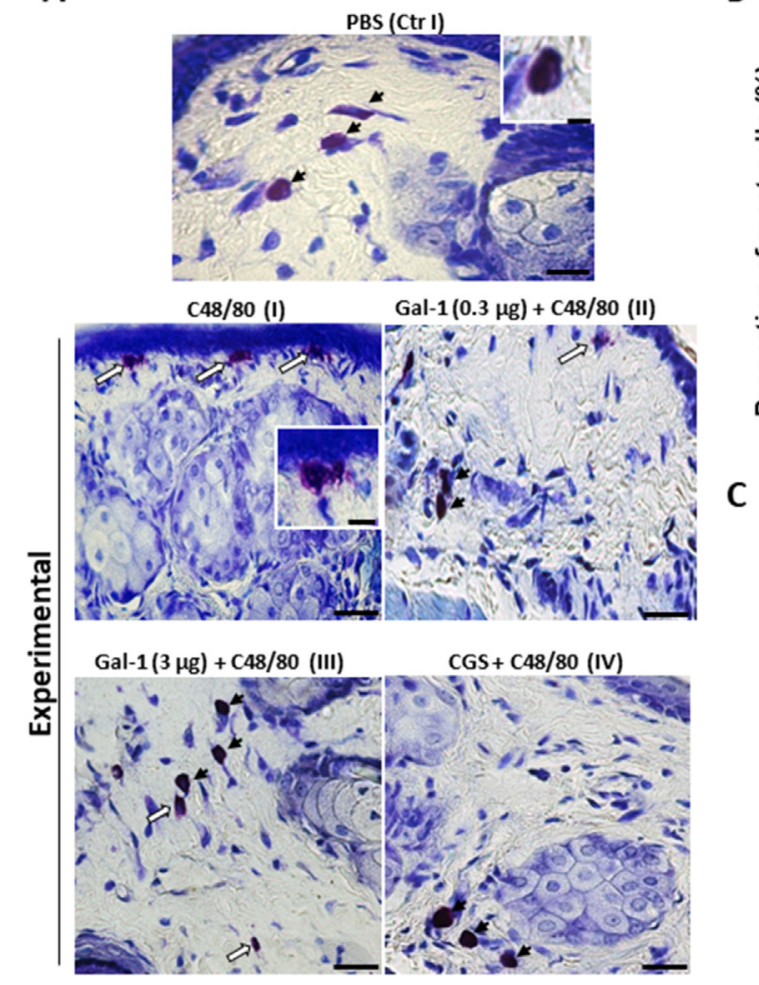

B
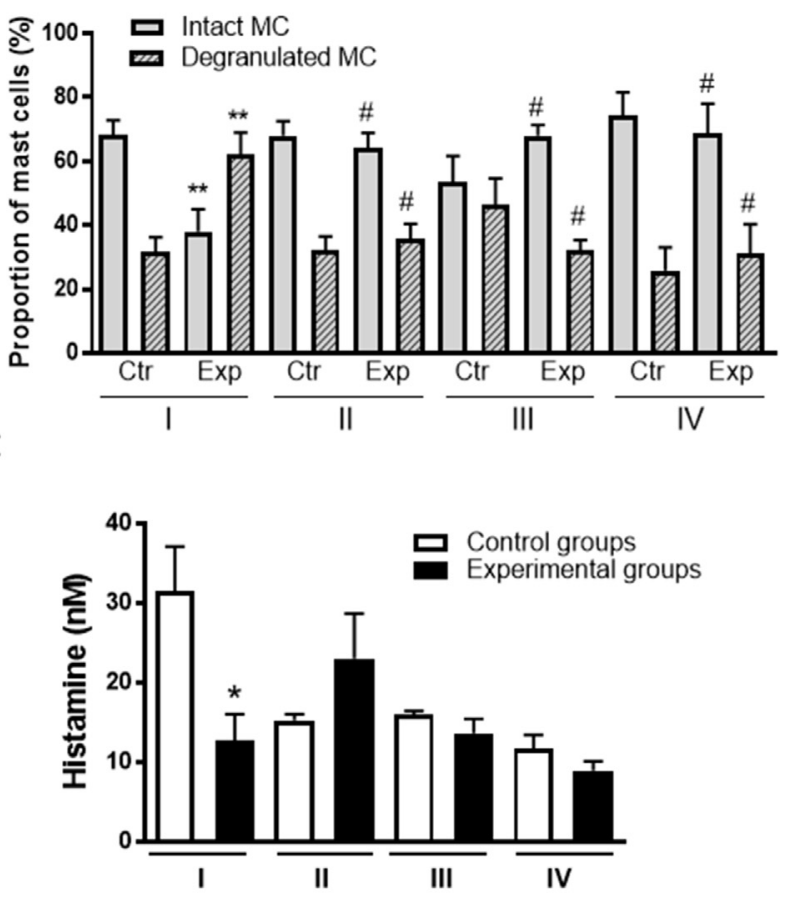

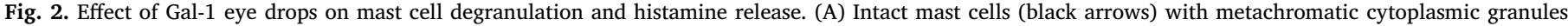

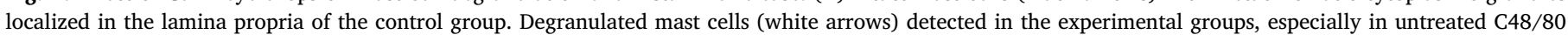

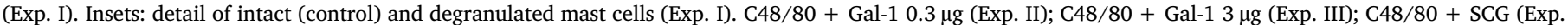

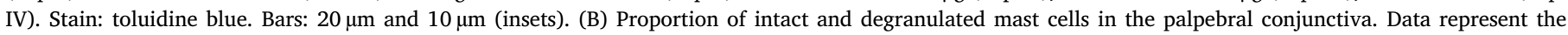

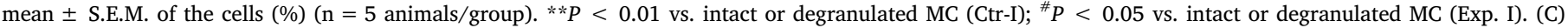

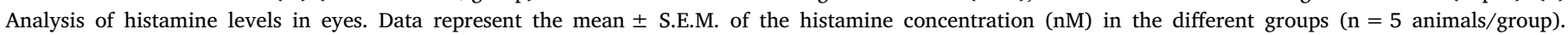
${ }^{* *} P<0.01,{ }^{* * *} P<0.001$ vs. corresponding control groups; ${ }^{\# \#} P<0.001$ vs. C48/80 (Exp. I).

II), Gal-1 plus C48/80 increased ocular levels of IFN- $\gamma$, IL- 6 and IL-10 in relation to the respective control (II) and untreated C48/80 groups (Exp. I) (Fig. 4). Interestingly, high concentration Gal-1 (3 $\mu$ g) and SCG (Exp. III and IV, respectively) decreased IFN- $\gamma$ and IL-4 levels (Fig. 4), confirming their local anti-inflammatory effects. All experimental groups (I-IV) showed a significant increase in IL-6 levels relative to the controls (Fig. 4).

Considering that MAPKs (a well-conserved signalling pathway) have been implicated in inflammatory responses contributing to leukocyte recruitment and cytokine production (Arthur and Ley, 2013), we performed Western blot analysis to understand the downstream molecular signalling pathways involved in the effect of treatment with Gal-1 eye drops in conjunctivitis. The phosphorylation of ERK, JNK and p38 MAPKs was assessed in the pooled eye extracts $(n=3$ animals per group) from different experimental conditions. C48/80-challenged eyes (Exp. I) exhibited increased levels of p-p38, p-JNK e p-ERK (Fig. 5) in relation to their respective control group I. Pharmacological treatments attenuated the C48/80 effect as evidenced by decreased expression of phosphorylated protein kinases, especially for Gal-1 at $3 \mu \mathrm{g}$ and SCG. As expected, control groups I to IV demonstrated weak immunoreactivity for MAPKs.

\section{Discussion}

In the eye, the anti-inflammatory role of Gal-1 has been evidenced in experimental models of corneal infections induced by Herpes simplex and Pseudomonas aeruginosa and uveitis (Rajasagi et al., 2012; Romero et al., 2006; Suryawanshi et al., 2013; Toscano et al., 2006; Zanon et al., 2015). Some of these studies showed that systemic or local administration of Gal-1 decreased influx of leukocyte and proinflammatory cytokine release, ameliorating ocular disease (Rajasagi et al., 2012; Suryawanshi et al., 2013; Toscano et al., 2006; Zanon et al., 2015). In addition, Gal-1 is a pro-angiogenic molecule able to bind to vascular endothelial growth factor receptor 2 (VEGFR2) in fibrovascular tissues in the eyes of diabetic retinopathy patients, leading to angiogenesis signalling pathway (Kanda et al., 2015). In this regard, silencing of Gal1 with adenoviral-Gal-1-RNA interference represent a promising strategy for the treatment of retinal neovascularization diseases (Yang et al., 2017). Here, we demonstrate that Gal-1 regulates the activation of mast cells and subsequent ocular allergy through macroscopic, histological, biochemical and molecular analyses using a model of experimental conjunctivitis induced by $\mathrm{C} 48 / 80$.

The efficacy of our experimental model was confirmed by the clinical scores after $20 \mathrm{~min}$ of C48/80 ocular instillation. As expected, eyelid oedema, conjunctiva and tearing were associated with a significant increase in the proportion of degranulated mast cells in the palpebral conjunctiva treated with C48/80 compared to the control group. Furthermore, a marked decrease of histamine in eye homogenates after $6 \mathrm{~h}$ of C48/80 challenge, confirmed the rapid activation of mast cells and release of their pre-stocked mediators. Consistently, other studies using this C48/80-induced conjunctivitis model detected a decrease in histamine levels in conjunctiva homogenates after $45 \mathrm{~min}$, 1, 12 and 24 h (Giannoulaki et al., 2003; Papathanassiou et al., 2011; Tiligada et al., 2000).

In addition, we detected an intense influx of eosinophils in the rat palpebral conjunctiva after $\mathrm{C} 48 / 80$ challenge, which was corroborated by the intense immunoreactivity of EPX in the eye homogenates. The importance of mast cells in the induction of neutrophil and eosinophil transmigration has been demonstrated in models of acute (Ajuebor et al., 1999), systemic (Da Silva et al., 2011) and allergic inflammation 
A
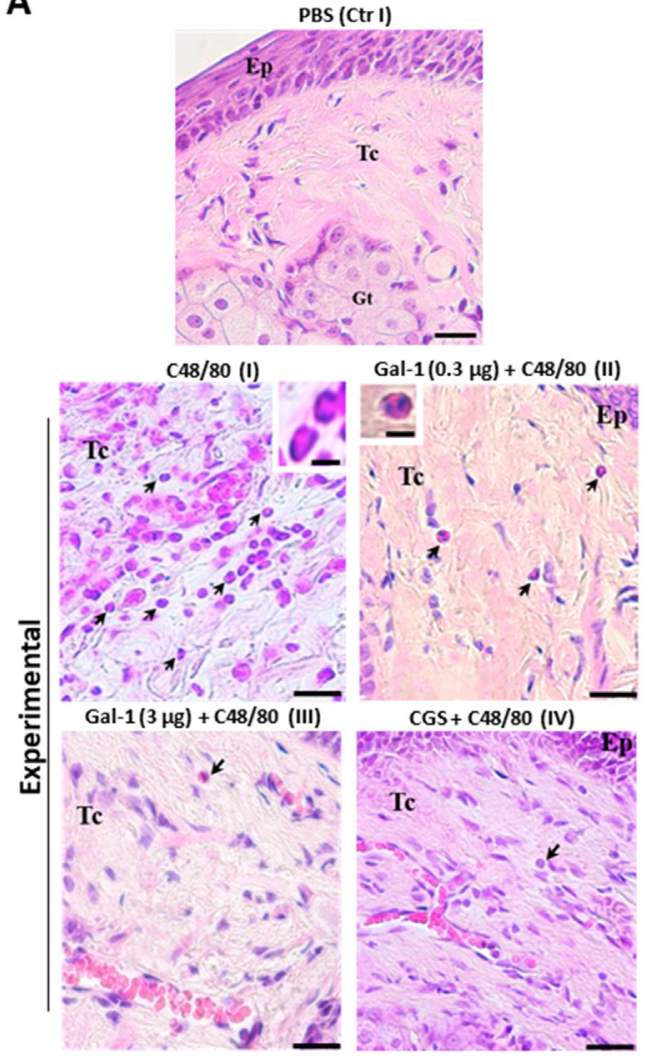

B
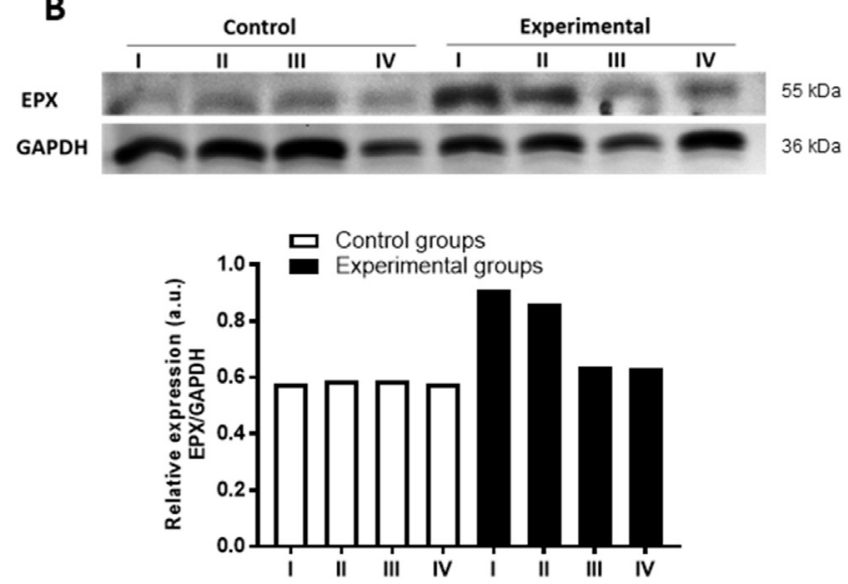

C

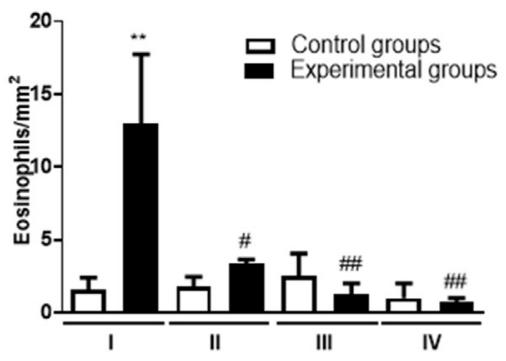

Fig. 3. Gal-1 eye drops regulate eosinophil influx into the palpebral conjunctiva. (A) A marked influx of eosinophils (arrows) was detected in the palpebral conjunctiva $6 \mathrm{~h}$ after C48/80 ocular instillation (Exp. I) compared to control, Gal-1- and SCG-treated groups (Exp. II-IV). Eyelids of the control group I (PBS) exhibited conjunctiva with normal aspect. Stratified squamous epithelium (Ep). Loose connective tissue (Tc). Blood vessel (V). Tarsal gland (Gt). Insets: detail of the migrated eosinophils (Exp. I and II). Stain: haematoxylin-eosin. Bars: $20 \mu \mathrm{m}$ and $10 \mu \mathrm{m}$ (insets). (B) Expression of eosinophil peroxidase (EPX) in the eye homogenates. C48/80challenged eyes (Exp. I) exhibited strong immunoreactivity for EXP (55 kDa) compared to the other groups. Immunoreactive bands were semiquantified by densitometry and expressed as arbitrary units relative to GAPDH (36 kDa; protein loading control). The data illustrate one representative example of two independent experiments. (C) Quantification of eosinophils. Data represent the mean \pm S.E.M. of the number of cells per $m^{2}{ }^{2}$ ( $\mathrm{n}=5$ animals $/$ group). ${ }^{* * P}<0.01$ vs. Control Group I; ${ }^{\#} P<0.05 ;{ }^{\# \#} P<0.01$ vs. Experimental - Group I.

(Fukuda et al., 2009; Miyazaki et al., 2008). Two of these studies, using selective depletion of mast cells induced by C48/80, showed a decreased influx of neutrophils into the peritoneal cavity after $4 \mathrm{~h}$ of zymosan-induced peritonitis (Ajuebor et al., 1999), and into the aqueous humour after $24 \mathrm{~h}$ of endotoxin-induced uveitis (Da Silva et al., 2011), compared to animals with non-depleted mast cells. Furthermore, in a model of pollen-induced AC, mast cell-deficient mice did not exhibit clinical signs and eosinophilia in the conjunctiva as severe as in the wild-type animals. This effect was reversed when deficient animals were repopulated with mast cells ( Fukuda et al., 2009; Miyazaki et al., 2008). These results show that mast cells are essential in inducing the initial response to allergic conjunctivitis (clinical signs) and recruitment of eosinophils.

Pharmacological treatment with SCG and both Gal-1 concentrations ( 0.3 and $3 \mu \mathrm{g} /$ eye) abrogated the effect of C48/80 challenge by reducing clinical signs of conjunctivitis, mast cell degranulation and eosinophilia, as well as the expression of EPX. Our findings reinforce the important regulatory role of Gal-1 in mast cell activation and consequent inhibition of ocular inflammation. In fact, SCG is a nonsteroidal anti-inflammatory drug traditionally described as an inhibitor of mast cell degranulation, and consequent release of histamine and other inflammatory mediators (Owen et al., 2004). In relation to Gal-1, recent studies have demonstrated that lack of endogenous Gal-1 in mice causes a greater influx of eosinophils in bronchoalveolar fluid and lung after induction of asthma by OVA (Ge et al., 2016). In vitro, Gal-1 administration reduced eotaxin-induced migration of eosinophils, which is independent of increased adhesion of these cells to the VCAM-1 adhesion molecule (Ge et al., 2016). Additionally, studies using OVAinduced AC mouse model showed that systemic Gal-1 treatment reduced clinical signs, Th2 (IL-4 and IL-13), eotaxin and RANTES levels in the lymph nodes $24 \mathrm{~h}$ after the final OVA challenge and compared with the untreated group (Mello et al., 2015).

Another interesting aspect detected in our rat model was the marked increase of IFN- $\gamma$, IL- 6 and IL-10 production in the eyes after treatment with Gal-1 $(0.3 \mu \mathrm{g})$ compared to the untreated C48/80. The cytokines IFN- $\gamma$ and IL- 6 can stimulate mast cell activation (Desai et al., 2016; Yu et al., 2011), while IL-10 inhibits mast cell activation (Bundoc and Keane-Myers, 2007). In fact, IFN- $\gamma$-deficient mice show a significant reduction in the clinical signs of conjunctivitis, asthma and the influx of leukocytes to tissues compared to wild-type animals (Yu et al., 2011). In vitro assays have also shown that IFN- $\gamma$ is able to activate mast cells, previously sensitized with IgE antibodies, inducing the release of histamine, IL- 6 and IL-13 after 1 or $24 \mathrm{~h}$ of challenge with specific antigens (Yu et al., 2011). On the other hand, IL-10-deficient mice exhibit reduced numbers of intact mast cells after administration of C48/80, an effect reversed by the administration of recombinant IL-10 (Bundoc and Keane-Myers, 2007). In addition, administration of recombinant IL-10 in an OVA-induced allergic rhinitis model reduced the influx of eosinophils and mast cells into the nasal mucosa, as well as reducing IL-5 and IL-17 in the nasal lavage fluid (Wang et al., 2014).

In contrast, ocular instillation of higher concentration Gal-1 ( $3 \mu \mathrm{g})$ and SCG in rat eyes decreased IL-4 levels in relation to the untreated C48/80 group. Similarly, in murine AC, systemic treatment with Gal-1 decreased IL-4 and IL-13 levels in lymph nodes $4 \mathrm{~h}$ after the final OVA 

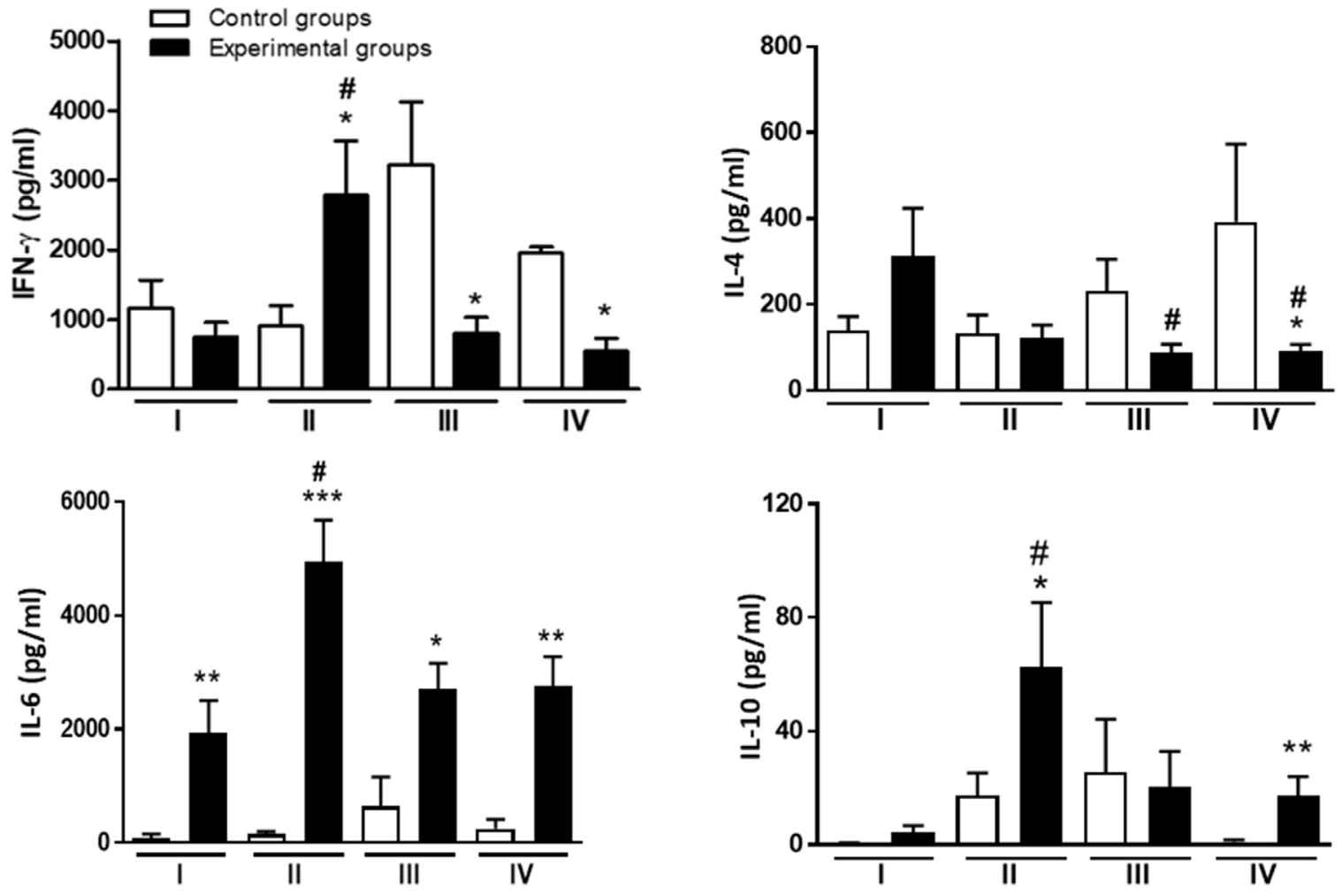

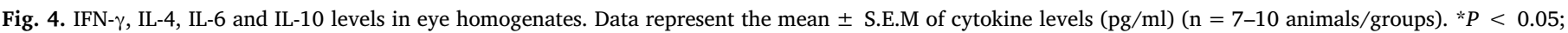
${ }^{* *} P<0.1 ;{ }^{* *} P<0.001$ vs. corresponding control groups; ${ }^{*} P<0.05$ vs. C48/80 (Exp. I).

challenge (Mello et al., 2015). Thus, the reduction of IL-4 levels may be associated with the ability of Gal-1 to inhibit the degranulation of mast cells producing this cytokine (Komiyama et al., 2014), regulating the Th2 lymphocyte response and IgE production. Furthermore, our study also shows that Gal-1 at $3 \mu \mathrm{g}$, but not at $0.3 \mu \mathrm{g}$, and SCG eye drops produced a pronounced decrease in phosphorylated p38, JNK and ERK kinase levels in eye homogenates compared to the untreated C48/80 group. MAPKs are crucial to induce the expression of multiple genes that together regulate the immune response (Arthur and Ley, 2013), our data suggests that the higher concentration of Gal-1 eye drops is more effective in controlling the inflammatory response induced by C48/80.

Overall, our findings show that pharmacological treatment by ocular instillation of Gal-1 has a potent immunomodulatory effect in C48/80-induced conjunctivitis, indicating that this lectin is an important therapeutic tool in ocular allergy.

\section{Acknowledgments}

The authors thank Fundação de Amparo à Pesquisa do Estado de São Paulo (FAPESP - grant numbers 2015/09858-3; 2016/02012-4) and the Conselho Nacional de Desenvolvimento Científico e Tecnológico (CNPq - grant number 308144/2014-7). CMB and ADG were PhD fellows from the Coordenação de Aperfeiçoamento de Pessoal de Nível Superior (CAPES).

\section{Conflict of interest}

The authors declare no conflict of interest in relation to the content of this article.
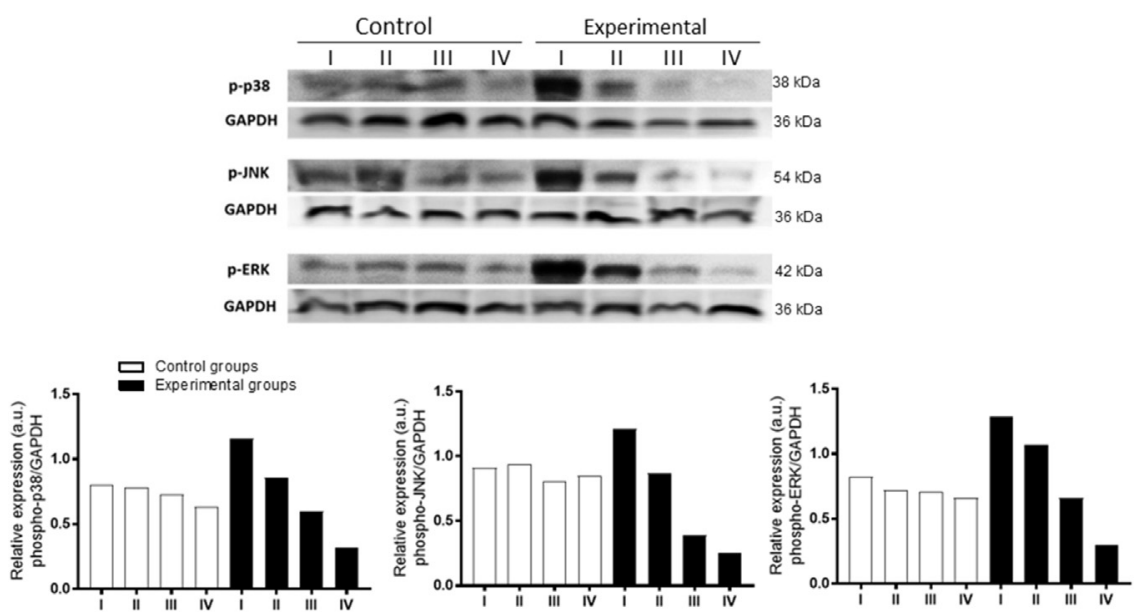

Fig. 5. Effect of Gal-1 eye drops on MAPK levels in eye homogenates. C48/80-challenged eyes (Exp. I) exhibited strong immunoreactivity for p-p38 (38 kDa), p-JNK (54 kDa) and p-ERK (42 kDa) compared to the other groups. Immunoreactive bands for MAPKs were semiquantified by densitometry and are expressed as arbitrary units of the ratio of p-MAPK/GAPDH (data illustrate one representative example of two independent experiments). GAPDH (36 kDa) was used as loading control. 


\section{Author contributions}

CMB and ADG performed the experiments. CMB, ADG, SMO and CDG contributed to the data analysis/interpretation. CMB and CDG wrote the manuscript. All authors have been reviewed and approved the final version of manuscript. CDG conceived and designed the study.

\section{References}

Ackerman, S., Smith, L.M., Gomes, P.J., 2016. Ocular itch associated with allergic conjunctivitis: latest evidence and clinical management. Ther. Adv. Chronic Dis. 7 , $52-67$.

Ajuebor, M.N., Das, A.M., Virág, L., Flower, R.J., Szabó, C., Perretti, M., 1999. Role of resident peritoneal macrophages and mast cells in chemokine production and neutrophil migration in acute inflammation: evidence for an inhibitory loop involving endogenous IL-10. J. Immunol. 162, 1685-1691.

Arthur, J.S., Ley, S.C., 2013. Mitogen-activated protein kinases in innate immunity. Nat. Rev. Immunol. 13, 679-692.

Blaser, C., Kaufmann, M., Müller, C., Zimmermann, C., Wells, V., Mallucci, L., Pircher, H., 1998. Beta-galactoside-binding protein secreted by activated T cells inhibits antigeninduced proliferation of T cells. Eur. J. Immunol. 28, 2311-2319.

Bucolo, C., Campana, G., Spadaro, A., Mangiafico, S., 1993. Effects of Mipragoside on ocular allergic inflammation in the rabbit. J. Ocul. Pharmacol. 9, 321-332.

Bundoc, V.G., Keane-Myers, A., 2007. IL-10 confers protection from mast cell degranulation in a mouse model of allergic conjunctivitis. Exp. Eye Res. 85, 575-579.

Da Silva, P.S., Girol, A.P., Oliani, S.M., 2011. Mast cells modulate the inflammatory process in endotoxin-induced uveitis. Mol. Vis. 17, 1310-1319.

Desai, A., Jung, M.Y., Olivera, A., Gilfillan, A.M., Prussin, C., Kirshenbaum, A.S., Beaven, M.A., Metcalfe, D.D., 2016. IL-6 promotes an increase in human mast cell numbers and reactivity through suppression of suppressor of cytokine signaling 3. J. Allergy Clin. Immunol. 137, 1863-1871 (e6).

Elieh Ali Komi, D., Rambasek, T., Bielory, L., 2018. Clinical implications of mast cell involvement in allergic conjunctivitis. Allergy 73, 528-539.

Fukuda, K., Ohbayashi, M., Morohoshi, K., Zhang, L., Liu, F.T., Ono, S.J., 2009. Critical role of IgE-dependent mast cell activation in a murine model of allergic conjunctivitis. J. Allergy Clin. Immunol. 124, 827-833 (e2)

Ge, X.N., Ha, S.G., Greenberg, Y.G., Rao, A., Bastan, I., Blidner, A.G., Rao, S.P., Rabinovich, G.A., Sriramarao, P., 2016. Regulation of eosinophilia and allergic airway inflammation by the glycan-binding protein galectin-1. Proc. Natl. Acad. Sci. USA 113, E4837-E4846.

Giannoulaki, V., Papathanassiou, M., Sitaras, N.M., Tiligada, E., 2003. Nadroparine inhibits the hypersensitivity response in the conjunctiva. Eur. J. Pharmacol. 481, 119-124.

Gil, C.D., Cooper, D., Rosignoli, G., Perretti, M., Oliani, S.M., 2006a. Inflammation-induced modulation of cellular galectin-1 and-3 expression in a model of rat peritonitis. Inflamm. Res. 55, 99-107.

Gil, C.D., Gullo, C.E., Oliani, S.M., 2011. Effect of exogenous galectin-1 on leukocyte migration: modulation of cytokine levels and adhesion molecules. Int. J. Clin. Exp. Pathol. 4, 74-84.

Gil, C.D., La, M., Perretti, M., Oliani, S.M., 2006b. Interaction of human neutrophils with endothelial cells regulates the expression of endogenous proteins annexin 1, galectin1 and galectin-3. Cell Biol. Int. 30, 338-344.

Kanda, A., Noda, K., Saito, W., Ishida, S., 2015. Aflibercept traps galectin-1, an angiogenic factor associated with diabetic retinopathy. Sci. Rep. 5, 17946.

Ko, S.M., KIM, M.K., KIM, J.C., 2000. The role of nitric oxide in experimental allergic conjunctivitis. Cornea 19, 84-91.

Komiyama, H., Miyake, K., Asai, K., Mizuno, K., Shimada, T., 2014. Cyclical mechanical stretch enhances degranulation and IL-4 secretion in RBL-2H3 mast cells. Cell Biochem. Funct. 32, 70-76.

La, M., Cao, T.V., Cerchiaro, G., Chilton, K., Hirabayashi, J., Kasai, K., Oliani, S.M., Chernajovsky, Y., Perretti, M., 2003. A novel biological activity for galectin-1: inhibition of leukocyte-endothelial cell interactions in experimental inflammation. Am. J. Pathol. 163, 1505-1515.

Leffler, H., Carlsson, S., Hedlund, M., Qian, Y., Poirier, F., 2002. Introduction to galectins. Glycoconj. J. 19, 433-440.

Leonardi, A., Curnow, S.J., Zhan, H., Calder, V.L., 2006. Multiple cytokines in human tear specimens in seasonal and chronic allergic eye disease and in conjunctival fibroblast cultures. Clin. Exp. Allergy 36, 777-784

Magone, M.T., Chan, C.C., Rizzo, L.V., Kozhich, A.T., Whitcup, S.M., 1998. A novel murine model of allergic conjunctivitis. Clin. Immunol. Immunopathol. 87, 75-84.

Mello, C.B., Ramos, L., Gimenes, A.D., Andrade, T.R., Oliani, S.M., Gil, C.D., 2015 Immunomodulatory effects of galectin- 1 on an IgE-mediated allergic conjunctivitis model. Investig. Ophthalmol. Vis. Sci. 56, 693-704.

Miyazaki, D., Tominaga, T., Yakura, K., Kuo, C.H., Komatsu, N., Inoue, Y., Ono, S.J., 2008. Conjunctival mast cell as a mediator of eosinophilic response in ocular allergy. Mol. Vis. 14, 1525-1532.

Norling, L.V., Perretti, M., Cooper, D., 2009. Endogenous galectins and the control of the host inflammatory response. J. Endocrinol. 201, 169-184.

Norling, L.V., Sampaio, A.L., Cooper, D., Perretti, M., 2008. Inhibitory control of endothelial galectin-1 on in vitro and in vivo lymphocyte trafficking. FASEB J. 22, 682-690.

Owen, C.G., Shah, A., Henshaw, K., Smeeth, L., Sheikh, A., 2004. Topical treatments for seasonal allergic conjunctivitis: systematic review and meta-analysis of efficacy and effectiveness. Br. J. Gen. Pract. 54, 451-456.

Papathanassiou, M., Giannoulaki, V., Zampeli, E., Tiligada, E., 2011. Effect of aminoguanidine on the conjunctival histamine and nitrite levels in experimental conjunctivitis. J. Ocul. Pharmacol. Ther. 27, 137-142.

Rabinovich, G.A., Daly, G., Dreja, H., Tailor, H., Riera, C.M., Hirabayashi, J., Chernajovsky, Y., 1999. Recombinant galectin-1 and its genetic delivery suppress collagen-induced arthritis via T cell apoptosis. J. Exp. Med. 190, 385-398.

Rabinovich, G.A., Iglesias, M.M., Modesti, N.M., Castagna, L.F., Wolfenstein-Todel, C., Riera, C.M., Sotomayor, C.E., 1998. Activated rat macrophages produce a galectin-1 like protein that induces apoptosis of T cells: biochemical and functional characterization. J. Immunol. 160, 4831-4840.

Rajasagi, N.K., Suryawanshi, A., Sehrawat, S., Reddy, P.B., Mulik, S., Hirashima, M., Rouse, B.T., 2012. Galectin-1 reduces the severity of herpes simplex virus-induced ocular immunopathological lesions. J. Immunol. 188, 4631-4643.

Romero, M.D., Muiño, J.C., Bianco, G.A., Ferrero, M., Juarez, C.P., Luna, J.D., Rabinovich, G.A., 2006. Circulating anti-galectin-1 antibodies are associated with the severity of ocular disease in autoimmune and infectious uveitis. Investig. Ophthalmol. Vis. Sci. 47, 1550-1556.

Santucci, L., Fiorucci, S., Rubinstein, N., Mencarelli, A., Palazzetti, B., Federici, B. Rabinovich, G.A., Morelli, A., 2003. Galectin-1 suppresses experimental colitis in mice. Gastroenterology 124, 1381-1394.

Sundblad, V., Morosi, L.G., Geffner, J.R., Rabinovich, G.A., 2017. Galectin-1: a jack-of-alltrades in the resolution of acute and chronic inflammation. J. Immunol. 199, 3721-3730.

Suryawanshi, A., Cao, Z., Thitiprasert, T., Zaidi, T.S., Panjwani, N., 2013. Galectin-1mediated suppression of Pseudomonas aeruginosa-induced corneal immunopathology. J. Immunol. 190, 6397-6409.

Tiligada, E., Aslanis, D., Delitheos, A., Varonos, D, 2000. Changes in histamine conten following pharmacologically-induced mast cell degranulation in the rat conjunctiva. Pharmacol. Res. 41, 667-670.

Toscano, M.A., Commodaro, A.G., Ilarregui, J.M., Bianco, G.A., Liberman, A., Serra, H.M., Hirabayashi, J., Rizzo, L.V., Rabinovich, G.A., 2006. Galectin-1 suppresses autoimmune retinal disease by promoting concomitant Th2- and T regulatory-mediated anti-inflammatory responses. J. Immunol. 176, 6323-6332.

Udell, I.J., Abelson, M.B., 1981. Animal and human ocular surface response to a topical nonimmune mast-cell degranulating agent (compound 48/80). Am. J. Ophthalmol. 91, 226-230.

Wang, S.B., Deng, Y.Q., Ren, J., Xiao, B.K., Liu, Z., Tao, Z.Z., 2014. Exogenous interleukin10 alleviates allergic inflammation but inhibits local interleukin-10 expression in a mouse allergic rhinitis model. BMC Immunol. 15, 9.

Yang, N., Zhang, W., He, T., Xing, Y., 2017. Silencing of galectin-1 inhibits retinal neovascularization and ameliorates retinal hypoxia in a murine model of oxygen-induced ischemic retinopathy. Exp. Eye Res. 159, 1-15.

Yu, M., Eckart, M.R., Morgan, A.A., Mukai, K., Butte, A.J., Tsai, M., Galli, S.J., 2011. Identification of an IFN- $\gamma /$ mast cell axis in a mouse model of chronic asthma. J. Clin. Investig. 121, 3133-3143.

Zanon, C., de, F., Sonehara, N.M., Girol, A.P., Gil, C.D., Oliani, S.M., 2015. Protective effects of the galectin-1 protein on in vivo and in vitro models of ocular inflammation. Mol. Vis. 21, 1036-1050.

Zuñiga, E., Rabinovich, G.A., Iglesias, M.M., Gruppi, A., 2001. Regulated expression of galectin-1 during B-cell activation and implications for T-cell apoptosis. J. Leukoc. Biol. 70, 73-79. 